\section{Field performance of hermaphrodite papaya plants obtained through molecular selection and micropropagation}

\author{
Emanuel Araya-Valverde ${ }^{1,2}$, Antonio Bogantes ${ }^{3}$, Andrea Holst ${ }^{4}$, \\ Cristian Vargas-Mora ${ }^{1}$, Luis Gómez-Alpízar ${ }^{4}$, Arturo Brenes ${ }^{4}$, \\ Elodia Sánchez-Barrantes ${ }^{1}$, Max Chavarría ${ }^{1,4}$ and Luis Barboza- \\ Barquero $^{4 *}$
}

Abstract: Most commercial papaya varieties segregate hermaphrodite and female plants. Growers normally select hermaphrodite plants in the field, due to market preferences. This requires planting multiple plants per site and later thinning of the females, distinguished by flower bud inspection. Micropropagation or the use of molecular markers are two possibilities to grow only hermaphrodite plants. Under the tropical conditions of this study, the field performance of hermaphrodite papaya plants developed by both of these methods was described. Moreover, a multiplex qPCR reaction was optimized. Hermaphrodite seedlings selected by molecular markers and plants obtained by micropropagation, had a lower slenderness ratio and initiated fruit production at a lower height than those selected by the conventional practice of the orchard. An analysis of flower types between the sex determination methods indicated that growing one hermaphrodite papaya plant per hole reduces the percentage of female-sterile flowers, resulting in fruit set at a lower trunk height and higher yields.

Keywords: Carica papaya, Real Time PCR, sex determination, Pococí, flower plasticity.

\section{INTRODUCTION}

Sex determination of papaya is important because markets prefer the hermaphrodite fruit shape, which tends to be pear-shaped or elongated. In contrast, fruits of female plants are rounder and have a larger central cavity (Fitch et al. 2005a, Salinas et al. 2018). Most commercial hybrids are gynodioecious and segregate at a 1:1 ratio for female and hermaphrodite plants. Therefore, to produce as much hermaphrodite fruit as possible is a concern for farmers since female plants will always appear in a commercial orchard. The common practice to increase the percentage of hermaphrodite papaya is to plant 3-4 nursery plants per spot and to thin out the females at flowering, when visual sex determination is possible. This practice means that additional fertilizer, pesticides and extra seedlings are needed, and more labor must initially be invested in the plantation.

The challenge for growing only hermaphrodite plants in the field has been met by using clonally propagated plants (Chan and Teo 2002, Fitch et al.
Crop Breeding and Applied Biotechnology 19(4), 420-427, 2019 Brazilian Society of Plant Breeding. Printed in Brazil http://dx.doi.org/10.1590/198470332019v19n4a59

\section{*Corresponding author: E-mail: luisorlando.barboza@ucr.ac.cr (iD) ORCID: 0000-0002-4140-6598}

Received: 05 April 2019 Accepted: 14 June 2019

\footnotetext{
${ }^{1}$ Centro Nacional de Innovaciones Biotecnológicas, CeNAT-CONARE, 1174-1200, San José, Costa Rica

${ }^{2}$ Instituto Tecnológico de Costa Rica, 1597050, Cartago, Costa Rica ${ }^{3}$ Instituto Nacional de Innovación y Transferencia de Tecnología Agropecuaria, 382-1007, San José, Costa Rica

${ }^{4}$ Universidad de Costa Rica, 11501-2060, San
} José, Costa Rica 
2005a, Fitch et al. 2005b, Salinas et al. 2018) and molecular markers (Deputy et al. 2002, Fitch et al. 2005a). The field performance of hermaphrodite plants resulting from the standard practice of female plant removal was compared by Fitch et al. (2005a) to that in all-hermaphrodite orchards obtained by clonal propagation (cuttings and micropropagation) and marker-assisted selection in the nursery stage. These authors observed that clonally propagated plants were more vigorous and produced flowers earlier at a lower height than seedling plants. Recently, Salinas et al. (2018) reported that molecularly sexed papaya plants grew faster and taller than those selected by conventional sex determination.

The use of molecular markers to predict male-hermaphrodite papaya plants was first described by Urasaki et al. (2002) and Deputy et al. (2002). Urasaki et al. (2002) described a 450-bp RAPD (Random Amplified Polymorphic DNA) fragment, named PSDM (Papaya Sex Determination Marker), which was converted to a SCAR (Sequence-Characterized Amplified Region) marker that amplified a 225-bp fragment. In another study, Deputy et al. (2002) generated a SCAR marker (W11) that only amplified products in hermaphrodite and male plants. They screened a larger population of 1937 seedlings derived from crosses of 'Rainbow' ' Kamiya' ('Laie Gold') and 'Rainbow' ' 'Kapoho' ('Poamoho Gold'). Plants predicted to be hermaphrodites were planted in the field and confirmed with a prediction accuracy of $99.2 \%$. This W11 marker was also used for papaya sex determination of different cultivars of dioecious and gynodioecious papaya genotypes (Chaturvedi et al. 2014). In Costa Rica, the W11 marker allowed sex determination of 1500 seedlings of the hybrid 'Pococi', where the observed female: hermaphrodite plant ratio did not differ from the expected 1:1 segregation (Saalau-Rojas et al. 2009). Other studies used RAPD to develop sex-linked markers in papaya (Niroshini et al. 2000, Chaves-Bedoya and Nuñez 2007). Several of the SCAR markers mentioned above were also used to develop non-PCR based techniques for sex determination in papaya, such as Fluorescence In Situ Hybridization (FISH) (Abreu et al. 2015) and Loop-mediated isothermal amplification (LAMP) (Hsu et al. 2012).

Interestingly, these SCAR markers were not tested for Real Time PCR (qPCR). Additionally, comparisons of the field performance of papaya plants selected by the multi-planting practice with that of hermaphrodite plants selected by molecular sex determination or with micropropagation have not been reported for sites other than Hawaii, USA (Fitch et al. 2005a, Fitch et al. 2005b) or Almería, Spain (Salinas et al. 2018). Studies of this nature for the hybrid 'Pococí' are not available either. Thus, the purpose of this study under tropical conditions was to compare the field performance of 'Pococi' hermaphrodite plants obtained by the conventional multi-planting practice to those resulting from marker-assisted selection and micropropagation. This study also compared the methods end-point PCR and qPCR for sex determination of the papaya hybrid 'Pococí. Finally, the effects of growing single plants selected by molecular sex determination or in-vitro propagation on flower plasticity were described.

\section{MATERIAL AND METHODS}

\section{Field experiments}

To test the growth and production of hermaphrodite plants derived from molecular markers and micropropagation, two independent field experiments were conducted at the same location and the performance compared with that of plants selected by the conventional sex determination method, by which four plants per hole are thinned to one. Experiments were carried out in Pococí, in the province of Limón, Costa Rica (lat 10 16' 34" N, long 83 47' 39" W, alt $125 \mathrm{~m}$ asl). The first experiment was conducted in 2016 and the second nine months later, in 2017. The average minimum temperature of the study area is $20^{\circ} \mathrm{C}$ and the average maximum $34{ }^{\circ} \mathrm{C}$. The average monthly rainfall during the time of the experiments was $302 \mathrm{~mm}$. In-vitro grown plants were provided by the plant biotechnology laboratory of the "Centro de Investigaciones Agronómicas" of the University of Costa Rica. Rooted plantlets were removed from the jars and gently washed under running tap water. Plantlets were transplanted into 72-well trays filled with TS1 growth medium (Klasmann-Deilmann $\mathrm{GmbH}$, Germany) and placed in a mist bed for 15 days before moving them to a shaded greenhouse. Later, they were moved to an unshaded greenhouse for one week prior to field planting. The molecular sex determination was performed as described in the following paragraphs.

\section{Plant material for sex determination}

Leaves of adult female and hermaphrodite papaya plants were sampled from the hybrid 'Pococi', which segregates for hermaphrodite and female plants in a 1:1 ratio. From ground leaf tissue $(50 \mathrm{mg})$, DNA was isolated using a micromortar 


\section{E Araya-Valverde et al.}

and a CTAB-based protocol (Doyle and Doyle 1990), with modifications, and per sample, $750 \mu \mathrm{L}$ of the following extraction buffer was added: 20 mM EDTA (pH 8.0); 100 mM Tris (pH 8.0); 2\% PVP, 2\% CTAB; $1.4 \mathrm{M} \mathrm{NaCl}$ and 0.2\% v/v $\beta$-mercaptoethanol. After incubation at $65^{\circ} \mathrm{C}$ for $20 \mathrm{~min}, 750 \mu \mathrm{L}$ of a chloroform:octanol solution (24:1) was added and mixed by inverting the tubes. The tubes were later centrifuged at 13,000 rpm for $5 \mathrm{~min}$, and $300 \mu \mathrm{L}$ of the supernatant was transferred into a new $1.5 \mathrm{~mL}$ tube, to which the same volume of cold isopropanol was added. After incubation for $5 \mathrm{~min}$ at room temperature, the tubes were centrifuged at $13000 \mathrm{rpm}$ for $5 \mathrm{~min}$. Isopropanol was discarded and the pellet washed by adding $500 \mu \mathrm{L}$ cold 70\% ethanol. After centrifugation at $13000 \mathrm{rpm}$ for $2 \mathrm{~min}$, the ethanol was discarted and the pellet dried at $37^{\circ} \mathrm{C}$. This pellet containing the DNA was resuspended by adding $50 \mu \mathrm{L}$ TE (Tris-EDTA) buffer and the DNA concentration was measured in a spectrophotometer NanoDrop (Thermo Scientific, USA), and finally

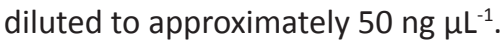

\section{Sex determination in adult plants by end-point PCR}

The first step consisted in the validation of two previously reported molecular markers to determine the sex of 'Pococí' papaya plants. For this purpose, multiplex end-point PCR reactions were performed with the primers W11-F ( $F$ for Forward) and W11-R ( $R$ for Reverse), T1-F and T1-R, described by Deputy et al. (2002). The PCR reactions had a final volume of $12 \mu \mathrm{L}$, containing DreamTaq buffer ( $1 \mathrm{X}$, ThermoScientific, USA), 0.625 units of DNA polymerase (ThermoScientific, USA), $0.2 \mathrm{mM}$ dNTPs, $0.25 \mu \mathrm{M}$ of each forward and reverse primer, and $100 \mathrm{ng}$ DNA isolated from adult female and hermaphrodite papaya plants of the hybrid 'Pococí'.

The PCR program consisted of an initial denaturalization step at $95^{\circ} \mathrm{C}$ for $5 \mathrm{~min}$, followed by 25 cycles at $95^{\circ} \mathrm{C}$ for $1 \mathrm{~min}$, $56^{\circ} \mathrm{C}$ for $1 \mathrm{~min}$ and $72^{\circ} \mathrm{C}$ for $1 \mathrm{~min}$, plus a final elongation step at $72^{\circ} \mathrm{C}$ for $7 \mathrm{~min}$. After PCR, loading dye (ThermoScientific, USA) was added to the samples to a concentration of $1 \mathrm{X}$ and electrophoresis performed on $1 \%$ agarose gel in TBE buffer.

Separately, the forward SDP-1 and reverse SDP-2 primers described by Urasaki et al. (2002) were also tested by end-point PCR. The final reaction volume of $25 \mu \mathrm{L}$ contained DreamTaq PCR Master Mix (1X, ThermoScientific, USA), $0.3 \mu \mathrm{M}$ of each forward and reverse primer and $\sim 100 \mathrm{ng}$ DNA isolated from adult female and hermaphrodite 'Pococi' plants. The PCR program consisted of an initial denaturalization step at $95^{\circ} \mathrm{C}$ for $5 \mathrm{~min}$, followed by 30 cycles at $95{ }^{\circ} \mathrm{C}$ for $1 \mathrm{~min}, 56^{\circ} \mathrm{C}$ for $1 \mathrm{~min}, 72{ }^{\circ} \mathrm{C}$ for $1 \mathrm{~min}$, and a final elongation step at $72{ }^{\circ} \mathrm{C}$ for $7 \mathrm{~min}$.

\section{Sex determination in adult plants by qPCR}

Reactions for qPCR were set up at $10 \mu \mathrm{L}$ of the final volume by incorporating Maxima SYBR Green Master Mix (1X, Thermo Scientific, USA) SDP-1 and SDP-2 primers (Urasaki et al. 2002) at $0.2 \mu \mathrm{M}$ each and DNA ( 100 ng). The DNA was the same as that used for end-point PCR. Amplification was performed in a 7500 PCR Real Time System (Applied Biosystems, USA) by a two-step protocol: $50{ }^{\circ} \mathrm{C}$ for $2 \mathrm{~min}$ and one initial denaturalization step at $95^{\circ} \mathrm{C}$ for $10 \mathrm{~min}$. Thermal cycling consisted of 28 cycles at $95^{\circ} \mathrm{C}$ for $15 \mathrm{~s}$, followed by an annealing and extension step at $56^{\circ} \mathrm{C}$ for $1 \mathrm{~min}$. After thermal cycling, the melting curve was determined at temperatures from $60^{\circ} \mathrm{C}$ to $95^{\circ} \mathrm{C}$, in $1{ }^{\circ} \mathrm{C}$ intervals. At each temperature, data were collected for $30 \mathrm{~s}$.

\section{Development of new primer sets and multiplex qPCR for sex determination}

The primers SDP-1 and SDP-2 developed by Urasaki et al (2002) were used to detect the hermaphrodite genotypes. In this study, the forward primer was modified (according to sequence no. AY428944.1 of the NCBI database) to contain an adenine at position nine (SDP-1 (modified) 5'-GCACGATTATAGATTAGATGT-3'). The following four primer sets were tested in multiplex assays together with the SDP primers: P01F 5'-GGTATGGAGATGCGACACAA-3' together with P01R 5'-ATTTCGCGCCTTTGACTCTT-3'; P02F 5'-GTTGCCCGATAAGCACTATGC-3' together with P02R 5'-AAGTGGAGGGAGAAGCTCAC-3'; P03F 5'-TCCTTCGGTTACTTCAAGCAG-3' together with P03R 5'- TGAGGCATTGTTGATGATCC-3'; and P04F 5'GCTGTTGGCCATTTTCATTT-3' together with P04R 5'-GGCCAAGCAGCTAGTTCAGA-3'. The primers were designed with software Primer BLAST and Primer 3 Plus (Untergasser et al. 2007). Each primer set was previously tested with the SDP primers to detect primer-dimer formation by the Multiple Primer Analyzer (Thermo Scientific, USA).

The multiplex qPCR reaction mix contained a final concentration of $1 X$ Maxima SYBR Green/ROX qPCR Master Mix (Thermo Scientific, USA). Each primer set was optimized to a final concentration of $0.075 \mu \mathrm{M}$ for P01F-R and P02F-R, 
combined with $0.3 \mu \mathrm{M}$ for SDP-1 and SDP-2. The DNA was extracted as mentioned above and $3 \mu$ lapproximately 30 to $60 \mathrm{ng} \mathrm{hL}^{-1}$ ) of it added. The final volume was $15 \mu \mathrm{L}$ (adjusted with DEPC-treated water). The method was tested on a set of 125 plants, derived from a selfed hermaphrodite plant, which were analyzed with primer set P01F-R, combined with SDP-1 and SDP-2; female, hermaphrodite and negative controls were included in every run.

The qPCR was performed in the Corbett Research RG 6000 thermocycler. Reactions were first heated at $95^{\circ} \mathrm{C}$ for 10 min, followed by $30 \mathrm{cycles}$ of $95^{\circ} \mathrm{C}$ for $30 \mathrm{~s}, 57^{\circ} \mathrm{C}$ for $60 \mathrm{~s}$ and $72^{\circ} \mathrm{C}$ for $30 \mathrm{~s}$. Fluorescence was measured in the extension step. Finally, melting curves were constructed by increasing the temperature from $50.5^{\circ} \mathrm{C}$ to $94.5^{\circ} \mathrm{C}$, at $1{ }^{\circ} \mathrm{C}$ per step. The melting temperature $(\mathrm{Tm})$ of each of the two amplification products was manually computed from the negative derivative of fluorescence versus temperature (-dF/dT) plot. Results were confirmed by $3 \%$ agarose (Top Vision, Thermo Scientific) gel electrophoresis (100 V, $1.5 \mathrm{~h})$.

\section{Comparison of sex prediction by end-point PCR and qPCR of nursery plants}

Two different sets of papaya plants in the nursery stage (second true leaf expanded) were analyzed. The first set consisted of 510 'Pococí' plants segregating in a 1:1 ratio for hermaphrodite and female plants. The second set was a 'Pococí' $F_{2}$ population of 385 plants, segregating in a 2:1 hermaphrodite:female plant ratio. Some modifications of the previously described DNA extraction method were included here. One leaf tip was placed in a $1.5 \mathrm{~mL}$ tube and ground in a Retsch Tissuelyser (26 1/s for 30 seconds, Retsch, Germany). The DNA was not individually quantified, instead, each isolation was diluted 10 times for qPCR and end-point PCR. End-point PCR was performed with the primers W11 and T1 (Deputy et al. 2002) and the PCR products visualized on agarose gels as described previously. The SDP primers (Urasaki et al. 2002) were used for qPCR. The final volume of $8 \mu \mathrm{L}$ of the qPCR mixture contained Maxima SYBR Green Master Mix (1.33X, Thermo Scientific, USA), $0.2 \mu \mathrm{M}$ of each of the primers SDP-1 and SDP-2 and 100 ng DNA. The qPCR program was the same as described in the previous section.

\section{Field validation of qPCR sex determination}

Plants of the said $\mathrm{F}_{2}$ population that had been classified as hermaphrodite by molecular markers, were sown in plant trays and maintained in a greenhouse for five weeks. Thereafter, 248 of these plants were transplanted to the field at a density of 1600 plants per hectare. The plant sex was visually assessed 8-10 weeks later when the flower buds can be optically examined. To validate the qPCR determination of hermaphrodites, we analyzed another set of 550 F1 nursery plants. The DNA was isolated from leaf tips and qPCR reactions and thermal cycling were performed under the above conditions. A total of 196 plants classified as hermaphrodites by the markers were transplanted to the field. Visual sex determination was carried out 8-10 weeks later as described above.-

\section{Data collection and analysis}

A randomized complete block design was used with four replications. The following model was tested: $Y i j=\mu+\tau i+$ $\beta j+\varepsilon i j$; where $\mu$ represents the mean; $\tau i$ the treatment effect, $\beta j$ the block effect; and $\varepsilon i j$ the experimental error. Each replicate contained 30 plants. Three treatments were tested: conventional sex determination (CSD), molecular sex determination (MSD) and in-vitro micro-propagated hermaphrodite (VMH) plants. For all treatments, seedlings were grown in plastic trays for six weeks and later planted in the field at a density of $2.0 \times 2.5 \mathrm{~m}$. For CSD, four plants were grown per planting hole, of which one hermaphrodite plant was selected, by visual inspection of the flower bud at the beginning of flowering, and the others were eliminated. Plant height and basal stem diameter were recorded monthly. Seven plants per replicate were evaluated for the first experiment and five for the second. Plot border plants were not taken into consideration. Plant height was measured from the ground to the apex and stem diameter at $10 \mathrm{~cm}$ above the ground with a vernier caliper. The slenderness ratio was calculated by dividing plant height by stem diameter. Height up to the first fruit was measured 200 days after transplanting (DAT). Female sterility was recorded during the second experiment. This trait was computed as percentage of the number of perfect (elongate) flowers in relation to the total number of flowers (perfect plus female-sterile flowers) per axil at 80, 97, 119 and 153 DAT.

The number of fruits at 200 and 198 DAT in experiments 1 and 2, respectively, was counted, as indicator of the potential yield. The fruits harvested at 278 and 295 DAT in experiments 1 and 2, respectively, were weighed and subjected to postharvest analysis. For fruit quality, six fruits per replicate from different plants were evaluated in the first and five 


\section{E Araya-Valverde et al.}

in the second experiment. Fruit skin firmness was measured in the middle of the fruit with a Chatillon mechanical force gauge (LG-200N), using a $10 \mathrm{~mm}$ diameter plunger. For pulp firmness, maximum values (expressed in Newtons, N) were reported. Fruits were cut longitudinally and the juice was extracted to determine fruit total soluble solids, expressed as ${ }^{\circ}$ Brix, with a refractometer (SPER Scientific, China).

Descriptive statistics were calculated and ANOVA tests performed with R software (R Core Team 2019). Post hoc Tukey tests were conducted using the R package Agricolae (De Mendiburu 2014).

\section{RESULTS AND DISCUSSION}

\section{Sex determination in adult plants by end-point and qPCR}

The end-point PCR assay resulted in the amplification of primer set T1 in all plants. Only hermaphrodite plants were amplified with the primer sets W11 (Deputy et al. 2002) and SDP (Urasaki et al. 2002). Both primer sets allowed the correct identification of hermaphrodite and female adult 'Pococi' plants. Amplification by qPCR with SDP primers was only observed in hermaphrodite adult plants with a cycle threshold of $22.94 \pm 0.49^{\circ} \mathrm{C}$. The melting curve revealed the presence of two amplicons at melting temperatures of $72.98 \pm 0.10^{\circ} \mathrm{C}$ and $81.12 \pm 0.10^{\circ} \mathrm{C}$.

\section{Comparison of sex prediction of nursery plants by end-point PCR and qPCR}

End-point PCR in F1 hybrid seeds ( $n=510)$ resulted in 270 hermaphrodite (amplification of both T1 and W11), 225 female (amplification of T1) and 15 unamplified samples. In the same set of samples, qPCR amplified 272 hermaphrodites with the SDP primer set, while 238 samples were not amplified and thus classified as female plants. Two hermaphrodites detected by qPCR contradicted the end-point PCR, which classified these samples as female. In further tests of the method by end-point PCR, a second set of 385 samples from F2 derived plants resulted in the amplification of 241 hermaphrodites (W11 and T1), 104 females (only T1) and 40 unamplified samples. The qPCR amplified for 260 hermaphrodites, while the remaining 125 samples did not amplify and were classified as female plants. In this set of samples, two mismatches between the methods were observed. The hermaphrodite:female segregation ratio was chi-square tested in both sets of plants. The first set was the $F_{1}$ hybrid $(n=510)$ with a theoretical segregation ratio of 1:1, while the second was the $F_{2}$ population $(n=385)$, with a theoretical ratio of 2:1. These tests confirmed that the observed agreed with the theoretical values, with $X^{2}$ values of $2.267(p=0.132)$ and $0.170(p=0.680)$, respectively.

The use of DNA markers to determine sex at the seedling stage is highly relevant when the selection of the economically important sex (hermaphrodite or female) has a direct impact on the agricultural business. In papaya, the SCAR markers W11 (Deputy et al. 2002) and SDP (Urasaki et al. 2002) amplify exclusively in hermaphrodite and male plants. The papaya sex-specific regions in the chromosomes of HSY (hermaphrodites) and its X counterpart in MSY (males) share a 4.6 Mbp region (Wang et al. 2012), which has facilitated the development of RAPD and SCAR markers that differentiate males and hermaphrodites from females (Liao et al. 2017). None of the SCAR markers developed for papaya sex determination had been tested for qPCR. The amplicons of qPCR are recommended to be $>200-b p$, with an ideal size between 80 and 120-bp (Thornton and Basu 2011). Consequently, we selected the SDP primer for qPCR amplification of DNA isolated from adult female and hermaphrodite plants, which has a 225-bp product. Our results show that the use of SDP primers (Urasaki et al. 2002) for qPCR using SYBR Green chemistry is a viable possibility to select hermaphrodite papaya plants.

\section{Field validation of sex determination with qPCR}

The 248 plants of the $F_{2}$ population selected as hermaphrodites based on molecular markers showed a prediction accuracy of $98.8 \%$ in the field. To validate the prediction of hermaphrodites, an additional $550 \mathrm{~F}_{1}$ plantlets were analyzed with the SDP primer set. The qPCR allowed the identification of 279 hermaphrodites and 271 females. This result agrees with the expected 1:1 segregation of hermaphrodite:female, with an $X^{2}$ value of $0.116(p=0.733)$. A total of 196 plants of this set reached the flowering stage in the field and visual examination indicated that 191 of them were in fact hermaphrodites, i.e., a $97.45 \%$ prediction success.

Marker W11 has been used for hermaphrodite prediction for the hybrid 'Pococi', at a success rate of 98\% (SaalauRojas et al. 2009). An accuracy rate of $99.2 \%$ with marker W11 was reported by Deputy et al. (2002) and of $99.6 \%$ by 
Fitch et al. (2005a). Errors during PCR set-up or field sampling errors are possible causes for the good but not $100 \%$ consistency. The use of other markers, e.g., Single Nucleotide Polymorphisms (SNP), seems promising to reach a $100 \%$ prediction accuracy, as recently described for cultivar BH-65 (Salinas et al. 2018).

\section{New primer sets for qPCR accurately predict sex of papaya plants}

Since the SDP primers only identify hermaphrodite genotypes, the reactions without amplification are regarded as females, which may however induce an error due to the DNA quality. Consequently, a multiplex reaction was conducted in a region of the papaya genome that would amplify for both female and hermaphrodite plants, together with the SDP primer that would amplify only for hermaphrodite plants. The melting curve analysis of the multiplex qPCR reactions showed two amplification products in the hermaphrodite samples in each of the four primer set combinations tested. In the female samples, only one amplification product was observed. The melting temperatures (Tm) of the SDP product were around $83^{\circ} \mathrm{C}$ in all tested reactions; the amplification product of the other primers (P01F-R, P02F-R) always had a lower Tm than the SDP product (Figure 1A, B). Products confirmed by melting analysis were checked by agarose gel electrophoresis, and the observed product sizes were as expected (296-bp for P01F-P01R, 160-bp for P02F-P02R, 154-bp for P03F-P03R, and 165-bp for P04F-P04R). For protocol validation, 76 hermaphrodite and 49 female plants were analyzed. This result agrees with the expected 2:1 segregation of hermaphrodite:female plants derived from a selfed hermaphrodite plant, with an $X^{2}$ value of $1.936(p=0.164)$. These results were confirmed with $100 \%$ accuracy at flowering in the field. In this study, we demonstrated that qPCR can be employed for sex determination in papaya using SDP primers. Additionally, a new reaction set of multiplex qPCR was developed to identify both hermaphrodite and female genotypes in the same reaction. An advantage of qPCR is that no agarose gels for DNA fragment separation are required, thus decreasing the cost per sample.

\section{Field performance}

For all studied traits, no significant effects were observed among the different blocks of the experiment $(P>0.05)$. The slenderness ratio (relation between plant height and diameter) was higher in the CSD than in the MSD and VMH plants in both experiments (Figure 1C, D). These differences were larger before flowering ( 60 days after transplanting). The MSD and VMH plants had larger stem diameters and lower heights than CSD plants. The thinner and taller stem observed in CSD was likely the result of strong competition for light in the early stages of plantlet development. In MSD and VMH plants, the height of the first fruit was lower than that of the CSD plants in both experiments (Figure 1E). The number of fruits was higher in MSD and VMH than CSD plants. Only in experiment 1 did MSD and CSD produce the same number of fruits (Table 1). Fruit weight was not significantly different between treatments in the first experiment but was highest in MSD and lowest in VMH plants in the second (Table 1). The MSD and VMH plants had higher yields than those of CSD in the second experiment, whereas these differences were not observed in the first. No significant differences were found for ${ }^{\circ}$ Brix or skin fruit firmness (P-value $>0.05$ ) among treatments in either experiment. Overall, total soluble solids averaged $12.0 \pm 0.9^{\circ} \mathrm{Brix}$ in experiment 1 , and $9.7 \pm 1.1^{\circ} \mathrm{Brix}$ in experiment 2 , whereas skin firmness averaged $128 \pm 54 \mathrm{~N}$ in experiment 1 and $115 \pm 44 \mathrm{~N}$ in experiment 2 .

Table 1. Number of fruit, fruit weight and fruit yield of papaya plants grown in three different systems. The number of fruits of experiment 1 and 2 was quantified 200 and 198 days after transplanting, respectively. Means and standard deviations from the mean (number in brackets) are shown. The letters indicate the results of post hoc Tukey's HSD test (for each experiment separately); means with different letters are significantly different $(P<0.05)$. Abbreviations: CSD, conventional sex determination; MSD, molecular sex determination; $\mathrm{VMH}$, in-vitro micro-propagated hermaphrodite plants

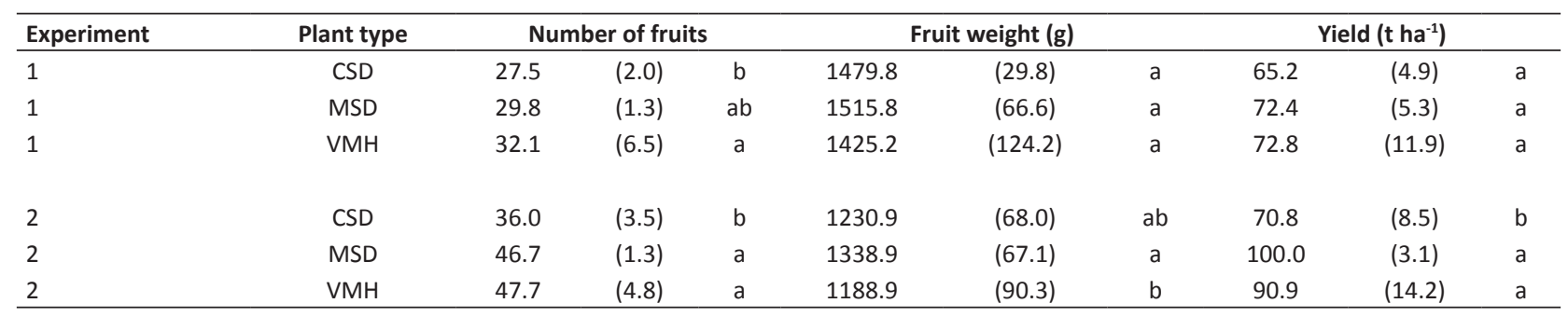




\section{E Araya-Valverde et al.}

This study is in agreement with Chan and Teo (2002) and Salinas et al. (2018), who observed that the papaya fruit quality of same-sex plants selected or produced by different sex determination systems does not differ. The effect of sex determination systems on yield, however, was not clear. In the case of fruit number, weight and yield, our study shows that the results differed between experiments, suggesting that other factors, such as weather or field conditions, may influence the results. According to Fitch et al. (2005b), yield traits are significantly influenced $(p<0.001)$ by harvest month and location. The cumulative yields varied drastically among locations, which was attributed to environmental differences. Slightly higher yields of seed-derived than of micro-propagated plants were reported by Talavera et al. (2007). In contrast, Chan and Teo (2002) did not observe such differences, while Salinas et al. (2018) reported a higher yield for plants selected by molecular markers, although apparently these results were statistically not different. An important point however, is that the conventional method of selecting hermaphrodites inevitably results in a 5-10\% loss of hermaphrodite plants in the field. In contrast, micropropagation as well as selection by molecular markers bypass this problem and are therefore advantageous, regardless of any other effect on plant yield per se.

During the first experiment, CSD plants had a higher degree of female sterility, as well as a longer and thinner stem. It was hypothesized that this led to a lower yield and a delay in fruit set, with fruit insertion at a higher point on the trunk. Therefore, in the second experiment, the slenderness ratio and a female sterility parameter were recorded in order to analyze these aspects in more detail. The conventional planting system induced sterility, which prevails long after flowering initiation (Figure 1F).

Our results indicate that hermaphrodite plants derived from molecular sex-determination as well as micropropagated hermaphrodite plants were shorter and produced fruit at a lower height than those established by the conventional practice, which requires transplanting multiple plants per site and selection of hermaphrodites at the flowering stage. These results are in agreement with Fitch et al. (2005a), who reported the same tendency and related these differences to a juvenility period, which was apparently either inexistent or shorter in the micropropagated plants. This is an important aspect when adult tissue is used for micro-propagation. However, our results also indicate that female sterility is significantly higher in plants grown in the conventional multi-plant system. These differences are particularly marked during the beginning of the reproductive phase (between 80 and 120 DAT, Figure $1 F)$, reducing the potential to produce fruit during this period. These differences might be explained by the fact that the conventional multi-planting method encourages competition for light between plants at an early age, resulting in different sink/source relationships that are unfavorable for the production of perfect flowers. Overall, the end result is a taller plant height and fruits are inserted higher
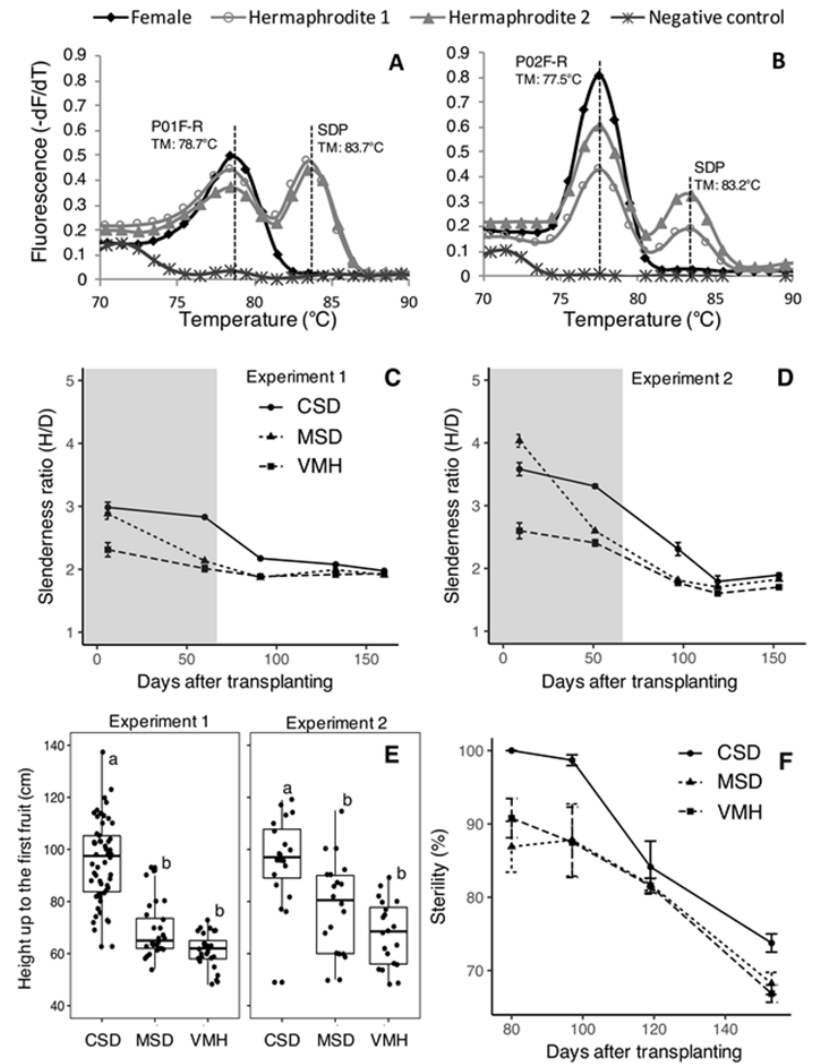

Figure 1. Dissociation curves of multiplex primer sets to determine the sex of papaya plants and field performance of hermaphrodite papaya obtained by different methods. The melting temperature $(\mathrm{Tm})$ of each amplified product is indicated on the vertical dotted line. A) Primer P01F-R with SDP, B) P02F-R with SDP. C, D) Slenderness ratio (height/diameter) of hermaphrodite papaya plants obtained by molecular selection and micropropagation, grown in two different experiments (Grey area indicates the vegetative growth phase prior to flowering). E) Height to the first fruit of hermaphrodite papaya plants obtained by molecular selection and micropropagation (letters indicate the results of the post hoc Tukey HSD test; means with different letters are significantly different, at $\mathrm{P}<0.05)$. F) Sterility of hermaphrodite papaya plants obtained by molecular selection and micropropagation. Sterility was calculated as the percentage of the total number of imperfect flowers. Mean values \pm standard error of the mean are shown. Abbreviations: CSD, conventional sex determination; MSD, molecular sex determination; $\mathrm{VMH}$, in-vitro micro-propagated hermaphrodite plants. 
on the trunk. These results are in contrast to those of Salinas et al. (2018), who compared the growth of molecular sex determined plants with the conventional multi-planting method. They claimed that molecular sex-determined plants were taller at first flowering and observed no differences regarding height of the first fruit. However, the study of Salinas et al. (2018) was conducted in a greenhouse with a dwarf variety, which may have reduced the competition among plants.

\section{ACKNOWLEDGMENTS}

The authors thank Eric Mora Newcomer for reviewing the manuscript. They also wish to thank Gabriela Palma and Felipe Rojas for technical assistance in the field experiments. The study was partially funded by FITTACORI (Fundación para el Fomento y Promoción de la Investigación y Transferencia de Tecnología Agropecuaria de Costa Rica) project F15-16. The authors declare that they have no conflict of interest.

\section{REFERENCES}

Abreu IS, Carvalho CR and Soares FAF (2015) Early sex discrimination in Carica papaya by nuclei FISH. Euphytica 206: 667-676.

Chan LK and Teo CKH (2002) Micropropagation of Eksotika, a Malaysian Papaya cultivar, and the field performance of the tissue culture derived clones. Acta Horticulturae 575: 99-105.

Chaturvedi K, Bommisetty P, Pattanaik A, Chinnaiyan V, M. Ramachandra D and Chennareddy A (2014) PCR Detection assay for sex determination in papaya using scar marker. Acta Botanica Croatica 73: 291-298.

Chaves-Bedoya G and Nuñez V (2007) A SCAR marker for the sex types determination in Colombian genotypes of Carica papaya. Euphytica 153: $215-220$

Deputy J, Ming R, Ma H, Liu Z, Fitch M, Wang M, Manshardt R and Stiles $\mathrm{J}$ (2002) Molecular markers for sex determination in papaya (Carica papaya L.). Theoretical and Applied Genetics 106: 107-111.

De Mendiburu F (2014) Agricolae: statistical procedures for agricultural research. R package version 1.2-1. Available at: <ftp://155.232.191.133/ cran/web/packages/agricolae/agricolae.pdf >. Accessed on January 2019.

Doyle JJ and Doyle JL (1990) Isolation of plant DNA from fresh tissue. Focus 12: 13-15

Fitch MMM, Moore PH, Leong TCW, Akashi LAY, Yeh AKF, White SA, De la Cruz AS, Santo LT, Ferreira SA and Poland LJ (2005a) Clonally propagated and seed-derived papaya orchards: I. Plant production and field growth. HortScience 40: 1283-1290.

Fitch MMM, Moore PH, Leong TCW, Akashi LAY, Yeh AKF, White SA, De la Cruz AS, Santo LT, Ferreira SA and Poland LJ (2005b) Clonally propagated and seed-derived papaya orchards: II. Yield comparisson. HortScience 40: 1291-1297.

Hsu T-H, Gwo J-C and Lin K-H (2012) Rapid sex identification of papaya (Carica papaya) using multiplex loop-mediated isothermal amplification (mLAMP). Planta 236: 1239-1246.

Liao Z, Yu Q and Ming R (2017) Development of male specific markers and identification of sex reversal mutants in papaya. Euphytica 213:53.

Niroshini E, Everard JMDT, Karunanayake EH and Tirimanne TLS (2000) Sex-specific random amplified polymorphic DNA (RAPD) markers in Carica papaya. Tropical Agriculture Research 12: 41-49.

R Core Team (2019) R: a language and environment for statistical computing. Vienna: R Foundation for Statistical Computing. Available at: < https://www.r-project.org>. Accessed on January 2019.

Saalau-Rojas E, Barrantes-Santamaría W, Loría-Quirós CL and BrenesAngulo A (2009) Identificación mediante PCR del sexo de la papaya (Carica papaya L.), híbrido 'Pococí'. Agronomía Mesoamericana 20: 311-317.

Salinas I, Salehi M, Hueso JJ and Cuevas S (2018) Assesment of two sexdetermining procedures in "BH-65" papaya from an economical and developmental point of view. Fruits 73: 184-197.

Talavera C, Espadas F, Contreras F and Santamaría JM (2007) Field performance of $100 \%$ hermaphrodite micropropagated papaya plants. Acta Horticultural 748: 219-222.

Thornton B and Basu C (2011) Real-time PCR (qPCR) primer design using free online software. Biochemistry and Molecular Biology Education 39: $145-154$

Untergasser A, Nijveen H, Rao X, Bisseling T, Geurts R and Leunissen JAM (2007) Primer3Plus, an enhanced web interface to Primer3. Nucleic Acids Research 35: W71-W74.

Urasaki N, Tokumoto M, Tarora K, Ban Y, Kayano T, Tanaka H, Oku H, Chinen I and Terauchi R (2002) A male and hermaphrodite specific RAPD marker for papaya (Carica papaya L.). Theoretical and Applied Genetics 104: 281285.

Wang J, Na J, Yu Q, Gschwend AR, Han J, Zeng F, Aryal R, VanBuren R, Murray JE, Zhang W, Navajas-Pérez R, Feltus FA, Lemke C, Tong EJ, Chen C, Wai CM, Singh R, Wang M-L, Min XJ, Alam M, Charlesworth D, Moore PH, Jiang J, Paterson AH and Ming R (2012) Sequencing papaya $\mathrm{X}$ and $\mathrm{Y} \mathrm{h}$ chromosomes reveals molecular basis of incipient sex chromosome evolution. Proceedings of the National Academy of Sciences 109: 13710-13715. 